\title{
ГИПЕРАКТИВАЦИЯ ИММУННОЙ СИСТЕМЫ ПРИ COVID-19 И НАРУШЕНИЯ ФУНКЦИИ ЩИТОВИДНОЙ ЖЕЛЕЗЫ. ЕСТЬ ЛИ СВЯЗЬ?
}

\author{
Колпакова Е.А., Айнетдинова А.Р., Никанкина Л.В., Трошина Е.А. \\ ФГБУ «НМИЦ эндокринологии» Минздрава России
}

\begin{abstract}
Среднетяжелое и тяжелое течение COVID-19 сопровождается гиперактивацией иммунной системы, вследствие чего можно ожидать усиления воздействия провоспалительных цитокинов на ткань щитовидной железы (ЩЖ) с последующим нарушением ее функции. Результаты проведенных корреляционных и сравнительных анализов полученных данных косвенно подтверждают гипотезу о повреждении щж при коронавирусной инфекции вследствие развития цитокинового шторма (ЦШ). Выявлена взаимосвязь уровней тиреотропного гормона (ТТГ) и гормонов ЩЖ и выживаемостью пациентов.
\end{abstract}

ЦЕЛЬ: выявление взаимосвязей уровней ТТГ, гормонов ЩЖ и маркеров воспалительного процесса, выживаемости госпитализированных пациентов с коронавирусной инфекцией.

МАТЕРИАЛЫ И МЕТОДЫ: в исследование были включены 122 пациента с установленным диагнозом при поступлении: «Коронавирусная инфекция, вирус идентифицирован/не идентифицирован. Внебольничная вирусная пневмония» Медиана возраста пациентов составила 59 лет [47; 72], соотношение мужчин/женщин 63/59 (52\%/48\%). Всем пациентам выполнены анализы крови на ТТГ, тироксин свободный (Т4св), трийодтиронин свободный (ТЗсв), интерлейкин-6 (ИЛ-6). Пациенты с развитием ЦШ (ИЛ-6>40 пг/мл) были разделены на две группы: в первой группе пациенты получали тоцилизумаб - peкомбинантное моноклональное антитело к человеческому рецептору ИЛ-6, во второй группе пациентам с ИЛ-6>40 пг/мл проводилась симптоматическая терапия основного заболевания.

РЕЗУЛЬТАТЫ: выполнен сравнительный анализ уровня ТТГ между группами выживших и умерших пациентов, госпитализированных в стационарные отделения ФБГУ НМИЦ эндокринологии МЗ РФ, перепрофилированные для лечения COVID-19. Медиана ТTГ у выживших пациентов составила 1,34 [0,85; 1,80], у умерших - 0,44 [0,29; 0,99]. Уровень госпитальной смертности был значимо выше у пациентов с низким уровнем ТТГ ( $p=0,012$ - критерий Манна-Уитни). Уровень ИЛ-6 статистически значимо коррелировал со значениями ТТГ (r: -0,221; p=0,024, метод Спирмена) и ТЗсв (r: -0,238; p=0,015 - метод Спирмена). На основании гипотезы, что иммуносупрессивный эффект генно-инженерной биологической терапии может оказывать протективную роль в отношении повреждения ЩЖ при развитии ЦШ был выполнен сравнительный анализ значений ТТГ в двух группах пациентов с ЦШ ( $p=0,080$ - критерий Манна-Уитни).

ВыВоды: у значительной части пациентов были обнаружены низкие значения ТТГ, что указывает на высокий риск развития тиреотоксикоза на фоне коронавирусной инфекции, по сравнению с популяцией. Низкие уровни ТТГ статистически значимо коррелировали с высокими значениями ИЛ-б. С одной стороны, эти изменения могут быть обусловлены гиперактивацией иммунной системы при COVID-19, с другой, с развитием синдрома эутиреоидной патологии при тяжелом течении болезни.

В пользу подтверждения гипотезы о повреждении ткани ЩЖ провоспалительными цитокинами говорят обнаруженные различия значений ТТГ на уровне статистической тенденции в 2 х группах пациентов с ЦШ, получавших терапию тоцилизумабом или симптоматическую терапию основного заболевания.

КЛЮЧЕВЫЕ СЛОВА: COVID-19; SARS-CoV-2; щитовидная железа; тиреопатии. 